\title{
Scatterings of Massive String States from D-brane and Their Linear Relations at High Energies
}

\author{
Chuan-Tsung Chan* \\ Department of Physics, Tung-Hai University, Taichung, Taiwan, R.O.C. \\ Jen-Chi Led ${ }^{\dagger}$ \\ Department of Electrophysics, National Chiao-Tung University, Hsinchu, Taiwan, R.O.C. \\ Yi Yang \\ Department of Electrophysics, National Chiao-Tung University and Physics Division, \\ National Center for Theoretical Sciences, Hsinchu, Taiwan, R.O.C.
}

(Dated: October 9, 2018)

\begin{abstract}
We study scatterings of bosonic massive closed string states at arbitrary mass levels from Dbrane. We discover that all the scattering amplitudes can be expressed in terms of the generalized hypergeometric function ${ }_{3} F_{2}$ with special arguments, which terminates to a finite sum and, as a result, the whole scattering amplitudes consistently reduce to the usual beta function. For the simple case of D-particle, we explicitly calculate high-energy limits of a series of the above scattering amplitudes for arbitrary mass levels, and derive infinite linear relations among them for each fixed mass level. The ratios of these high-energy scattering amplitudes are found to be consistent with the decoupling of high-energy zero-norm states of our previous works.
\end{abstract}

\footnotetext{
*Electronic address: ctchan@thu.edu.tw

${ }^{\dagger}$ Electronic address: jcclee@cc.nctu.edu.tw

${ }^{\ddagger}$ Electronic address: yyang@phys.cts.nthu.edu.tw
} 


\section{INTRODUCTION}

In contrast to the scattering of massless string states, massive higher-spin string scattering amplitudes [1] had not been well studied in the literature since the developement of string theory. Recently high-energy, fixed angle behavior of string scattering amplitudes [2, 3, 4] was intensively reinvestigated for massive string states at arbitrary mass levels [5, 6, 7, ㅇ, 9, 10, 11, 12, 13]. The motivation was to uncover the fundamental hidden stringy spacetime symmetry. An important new ingredient of this approach is the zero-norm states (ZNS) [1, 14, 15, 16] in the old covariant first quantized (OCFQ) string spectrum. One utilizes the decoupling of ZNS to obtain nonlinear relations or on-shell Ward identities among string scattering amplitudes. In the high energy limit, many simplications occur and one can derive linear relations among high-energy scattering amplitudes of different string states at each fixed but arbitrary mass levels. Moreover, these linear relations can be used to fix the ratios among high energy scattering amplitudes of different string states at each fixed mass level algebraically. This explicitly shows that there is only one independent component of high-energy scattering amplitude at each mass level. On the other hand, a saddle-point method was also developed to calculate the general formula of tree-level highenergy scattering amplitudes of four arbitrary string states to verify the ratios calculated above. This general formula expresses all high-energy string scattering amplitudes in terms of that of four tachyon as conjectured by Gross in 1988 [3].

In this paper, we study scatterings of bosonic massive closed string states at arbitrary mass levels from D-brane. The scattering of massless string states from D-brane was well studied in the literature and can be found in [17]. Since the mass of D-brane scales as the inverse of the string coupling constant $1 / g$, we will assume that it is infinitely heavy to leading order in $g$ and does not recoil. We will first show that, for the $(0 \rightarrow 1)$ and $(1 \rightarrow \infty)$ channels, all the scattering amplitudes can be expressed in terms of the beta functions, thanks to the momentum conservation on the D-brane. Alternatively, the Kummer relation of the hypergeometric function ${ }_{2} F_{1}$ can be used to reduce the scattering amplitudes to the usual beta function. After summing up the $(0 \rightarrow 1)$ and $(1 \rightarrow \infty)$ channels, we discover that all the scattering amplitudes can be expressed in terms of the generalized hypergeometric function ${ }_{3} F_{2}$ with special arguments, which terminates to a finite sum and, as a result, the whole scattering amplitudes consistently reduce to the usual beta function. Finally, for the 
simple case of D-particle, we explicitly calculate high-energy limit of a series of the above scattering amplitudes for arbitrary mass levels, and derive infinite linear relations among them for each fixed mass level. Since the calculation of decoupling of high energy ZNS remains the same as the case of scatterings without D-brane, the ratios of these high-energy scattering amplitudes are found to be consistent with the decoupling of high-energy zeronorm states of our previous works. [5, 6, 7, 8, 9, 10, 11, 12]. This paper is organized as follows. In section II, we first calculate closed string tachyon scatters to D-brane. The calculation is then generalized to arbitrary mass levels. By using the Kummer relation of the hypergeometric function ${ }_{2} F_{1}$, all the scattering amplitudes in the $(0 \rightarrow 1)$ and $(1 \rightarrow \infty)$ channels can be reduced to the usual beta function. We then sum up the $(0 \rightarrow 1)$ and $(1 \rightarrow \infty)$ channels, and show that all the scattering amplitudes can be expressed in terms of the terminated generalized hypergeometric function ${ }_{3} F_{2}$ with special arguments, and the whole scattering amplitudes consistently reduce to the usual beta function. In section III, for the simple case of D-particle, we explicitly calculate high-energy limit of a series of the above scattering amplitudes for arbitrary mass levels, and derive the linear relations among them. The results are compared to the calculations of decoupling of high energy ZNS. We give a brief conclusion in section IV. Some relevant formulas of the hypergeometric function ${ }_{2} F_{1}$ and the generalized hypergeometric function ${ }_{3} F_{2}$ are listed in the Appendix.

\section{SCATTERINGS OF MASSIVE STRING STATES FROM D-BRANE}

We first review some massive string scattering amplitudes for arbitrary mass levels without D-brane. The $(s, t)$ channel scattering amplitude of $26 \mathrm{D}$ open bosonic string with $V_{2}=\alpha_{-1}^{\mu_{1}} \alpha_{-1}^{\mu_{2}} \cdots \alpha_{-1}^{\mu_{n}}|0, k\rangle$, the highest spin state at mass level $M_{o p}^{2}=2(n-1)$, and three tachyons $V_{1,3,4}$ was calculated to be [12]

$$
\mathcal{T}_{n ; s t}^{\mu_{1} \mu_{2} \cdot \mu_{n}}=\sum_{l=0}^{n}(-)^{l}\left(\begin{array}{l}
n \\
l
\end{array}\right) B\left(-\frac{s}{2}-1+l,-\frac{t}{2}-1+n-l\right) k_{1}^{\left(\mu_{1}\right.} . . k_{1}^{\mu_{n-l}} k_{3}^{\mu_{n-l+1}} . . k_{3}^{\left.\mu_{n}\right)}
$$

where $B(u, v)=\int_{0}^{1} d x x^{u-1}(1-x)^{v-1}$ is the Euler beta function. The corresponding $(t, u)$ channel scattering amplitude can be calculated to be

$$
\mathcal{T}_{n ; t u}^{\mu_{1} \mu_{2} \cdot \mu_{n}}=\sum_{l=0}^{n}\left(\begin{array}{l}
n \\
l
\end{array}\right) B\left(-\frac{t}{2}+n-l-1,-\frac{u}{2}-1\right) k_{1}^{\left(\mu_{1}\right.} . . k_{1}^{\mu_{n-l}} k_{3}^{\mu_{n-l+1}} . . k_{3}^{\left.\mu_{n}\right)} .
$$


In calculating Eq.(2.2), we have used the Mobious transformation $y=(x-1) / x$ to change the integration region from $(1 \rightarrow \infty)$ to $(0 \rightarrow 1)$. One can see that all the scattering amplitudes above can be expressed in terms of the beta function.

In this section we will study the general structure of an arbitrary incoming closed string state scatters from D-brane and ends up with an arbitrary spin outgoing closed string states at arbitrary mass levels. In particular, we will examine whether they can be expressed in terms of beta function as the above scattering amplitudes without D-brane. We will first begin with the simple case of tachyon to tachyon scattering and then generalize to scatterings of states at arbitrary mass levels. The standard propagators of the left and right moving fields are

$$
\begin{aligned}
\left\langle X^{\mu}(z) X^{\nu}(w)\right\rangle & =-\eta^{\mu \nu} \log (z-w), \\
\left\langle\tilde{X}^{\mu}(\bar{z}) \tilde{X}^{\nu}(\bar{w})\right\rangle & =-\eta^{\mu \nu} \log (\bar{z}-\bar{w}) .
\end{aligned}
$$

In addition, there are also nontrivial correlator between the right and left moving fields as well

$$
\left\langle X^{\mu}(z) \tilde{X}^{\nu}(\bar{w})\right\rangle=-D^{\mu \nu} \log (z-\bar{w})
$$

as a result of the boundary condition at the real axis. Propagator Eq.(2.5) has the standard form Eq.(2.3) for the fields satisfying Neumann boundary condition, while matrix $D$ reverses the sign for the fields satisfying Dirichlet boundary condition. We will follow the standard notation and make the following replacement

$$
\tilde{X}^{\mu}(\bar{z}) \rightarrow D^{\mu}{ }_{\nu} X^{\nu}(\bar{z})
$$

which allows us to use the standard correlators Eq.(2.3) throughout our calculations. As we will see, the existence of the Propagator Eq.(2.5) has far-reaching effect on the string scatterings from D-brane.

\section{A. Tachyon to tachyon}

We first consider the tachyon to tachyon scattering amplitude 


$$
\begin{aligned}
A_{\text {tach }} & =\int d^{2} z_{1} d^{2} z_{2}\left\langle V_{1}\left(z_{1}, \bar{z}_{1}\right) V_{2}\left(z_{2}, \bar{z}_{2}\right)\right\rangle \\
& =\int d^{2} z_{1} d^{2} z_{2}\left\langle V\left(k_{1}, z_{1}\right) \tilde{V}\left(k_{1}, \bar{z}_{1}\right) V\left(k_{2}, z_{2}\right) \tilde{V}\left(k_{2}, \bar{z}_{2}\right)\right\rangle \\
& =\int d^{2} z_{1} d^{2} z_{2}\left\langle e^{i k_{1} X\left(z_{1}\right)} e^{i k_{1} \tilde{X}\left(\bar{z}_{1}\right)} e^{i k_{2} X\left(z_{2}\right)} e^{i k_{2} \tilde{X}\left(\bar{z}_{2}\right)}\right\rangle \\
& =\int d^{2} z_{1} d^{2} z_{2}\left(z_{1}-\bar{z}_{1}\right)^{k_{1} \cdot D \cdot k_{1}}\left(z_{2}-\bar{z}_{2}\right)^{k_{2} \cdot D \cdot k_{2}}\left|z_{1}-z_{2}\right|^{2 k_{1} \cdot k_{2}}\left|z_{1}-\bar{z}_{2}\right|^{2 k_{1} \cdot D \cdot k_{2}} .
\end{aligned}
$$

To fix the $S L(2, R)$ invariance, we set $z_{1}=i y$ and $z_{2}=i$. Introducing the $S L(2, R)$ Jacobian

$$
d^{2} z_{1} d^{2} z_{2}=4\left(1-y^{2}\right) d y
$$

we have, for the $(0 \rightarrow 1)$ channel,

$$
\begin{aligned}
A_{\text {tach }}^{(0 \rightarrow 1)} & =4(2 i)^{k_{1} \cdot D \cdot k_{1}+k_{2} \cdot D \cdot k_{2}} \int_{0}^{1} d y y^{k_{2} \cdot D \cdot k_{2}}(1-y)^{2 k_{1} \cdot k_{2}+1}(1+y)^{2 k_{1} \cdot D \cdot k_{2}+1} \\
& =4(2 i)^{2 a_{0}} \int_{0}^{1} d y y^{a_{0}}(1-y)^{b_{0}}(1+y)^{c_{0}} \\
& =4(2 i)^{2 a_{0}} \frac{\Gamma\left(a_{0}+1\right) \Gamma\left(b_{0}+1\right)}{\Gamma\left(a_{0}+b_{0}+2\right)}{ }_{2} F_{1}\left(-c_{0}, a_{0}+1, a_{0}+b_{0}+2,-1\right) \\
& =4(2 i)^{2 a_{0}} \frac{\Gamma\left(a_{0}+1\right) \Gamma\left(b_{0}+1\right)}{\Gamma\left(a_{0}+b_{0}+2\right)} 2^{-2 a_{0}-1-N}{ }_{2} F_{1}\left(N-a_{0}, b_{0}+1, a_{0}+b_{0}+2,-1\right) \\
& =4(2 i)^{2 a_{0}} 2^{-2 a_{0}-1-N} \int_{0}^{1} d t t^{b_{0}}(1-t)^{a_{0}}(1+t)^{a_{0}+N} .
\end{aligned}
$$

In the above calculations, we have defined

$$
\begin{aligned}
& a_{0}=k_{1} \cdot D \cdot k_{1}=k_{2} \cdot D \cdot k_{2}, \\
& b_{0}=2 k_{1} \cdot k_{2}+1, \\
& c_{0}=2 k_{1} \cdot D \cdot k_{2}+1,
\end{aligned}
$$

so that

$$
2 a_{0}+b_{0}+c_{0}+2=4 N_{1} \equiv-N
$$

and $-k_{1}^{2}=M^{2} \equiv \frac{M_{\text {closed }}^{2}}{2 \alpha_{\text {closed }}^{\prime}}=2\left(N_{1}-1\right), N_{1}=0$ for tachyon. We have also used the integral representation of the hypergeometric function

$$
{ }_{2} F_{1}(\alpha, \beta, \gamma, z)=\frac{\Gamma(\gamma)}{\Gamma(\beta) \Gamma(\gamma-\beta)} \int_{0}^{1} d t t^{\beta-1}(1-t)^{\gamma-\beta-1}(1-z t)^{-\alpha}
$$


and the following identity

$$
{ }_{2} F_{1}(\alpha, \beta, \gamma ; x)=(1-x)^{\gamma-\alpha-\beta}{ }_{2} F_{1}(\gamma-\alpha, \gamma-\beta, \gamma ; x)
$$

which we discuss in the appendix. In addition, the momentum conservation on the D-brane

$$
D \cdot k_{1}+k_{1}+D \cdot k_{2}+k_{2}=0
$$

is crucial to get the final result Eq.(2.10). Finally, by using change of variable $\tilde{t}=t^{2}$, Eq.(2.10) can be further reduced to the beta function

$$
A_{\text {tach }}^{(0 \rightarrow 1)} \simeq \frac{\Gamma\left(a_{0}+1\right) \Gamma\left(\frac{b_{0}+1}{2}\right)}{\Gamma\left(a_{0}+\frac{b_{0}}{2}+\frac{3}{2}\right)}=B\left(a_{0}+1, \frac{b_{0}+1}{2}\right)
$$

where we have omitted an irrelevant factor.

For the $(1 \rightarrow \infty)$ channel, we use the change of variable $y=\frac{1+t}{1-t}$ and end up with the same result

$$
\begin{aligned}
A_{\text {tach }}^{(1 \rightarrow \infty)} & =4(2 i)^{k_{1} \cdot D \cdot k_{1}+k_{2} \cdot D \cdot k_{2}} \int_{1}^{\infty} d y y^{k_{2} \cdot D \cdot k_{2}}(y-1)^{2 k_{1} \cdot k_{2}+1}(1+y)^{2 k_{1} \cdot D \cdot k_{2}+1} \\
& =4(2 i)^{2 a_{0}} 2^{-2 a_{0}-1-N} \int_{0}^{1} d t t^{b_{0}}(1-t)^{a_{0}+N}(1+t)^{a_{0}} \\
& \simeq \frac{\Gamma\left(a_{0}+1\right) \Gamma\left(\frac{b_{0}+1}{2}\right)}{\Gamma\left(a_{0}+\frac{b_{0}}{2}+\frac{3}{2}\right)}=B\left(a_{0}+1, \frac{b_{0}+1}{2}\right)
\end{aligned}
$$

since $N=0$ for the case of tachyon.

Altenatively, one can use the Kummer formula of hypergeometric function

$$
{ }_{2} F_{1}(\alpha, \beta, 1+\alpha-\beta,-1)=\frac{\Gamma(1+\alpha-\beta) \Gamma\left(1+\frac{\alpha}{2}\right)}{\Gamma(1+\alpha) \Gamma\left(1+\frac{\alpha}{2}-\beta\right)}
$$

and

$$
\Gamma\left(\frac{1+\alpha}{2}\right)=\frac{2^{-\alpha} \sqrt{\pi} \Gamma(1+\alpha)}{\Gamma\left(1+\frac{\alpha}{2}\right)},
$$

to reduce Eq.(2.9) to the final result Eq.(2.18) . In this calculation, we have used the Kummer condition

$$
\gamma=1+\alpha-\beta
$$

which is equivalent to the momentum conservation on the D-brane Eq.(2.17). 


\section{B. Tensor to tensor}

In this subsection, we generalize the previous calculation to general tensor to tensor scatterings. In this case, we define

$$
\begin{aligned}
& a=k_{1} \cdot D \cdot k_{1}+n_{a} \equiv a_{0}+n_{a} \\
& b=2 k_{1} \cdot k_{2}+1+n_{b} \equiv b_{0}+n_{b} \\
& c=2 k_{1} \cdot D \cdot k_{2}+1+n_{c} \equiv c_{0}+n_{c}
\end{aligned}
$$

where $n_{a}, n_{b}$ and $n_{c}$ are integer and

$$
N^{\prime}=-\left(2 n_{a}+n_{b}+n_{c}\right)
$$

so that

$$
2 a+b+c+2+N^{\prime}=4 N_{1} \Longrightarrow 2 a+b+c+2=4 N_{1}-N^{\prime} \equiv-N
$$

where $k_{1}^{2}=2\left(N_{1}-1\right)$ and $N_{1}$ is now the mass level of $k_{1}$. After a similar calculation as the previous subsection, it is easy to see that a typical term in the expression of the general tensor to tensor scattering amplitudes can be reduced to the following integral

$$
\begin{aligned}
I_{(0 \rightarrow 1)} & =\int_{0}^{1} d t t^{a}(1-t)^{b}(1+t)^{c}, \\
& =\frac{\Gamma(a+1) \Gamma(b+1)}{\Gamma(a+b+2)}{ }_{2} F_{1}(-c, a+1, a+b+2,-1) \\
& =2^{b+c+1} \frac{\Gamma(a+1) \Gamma(b+1)}{\Gamma(a+b+2)}{ }_{2} F_{1}(-a-N, b+1, a+b+2,-1) \\
& =2^{-2 a-1-N} \int_{0}^{1} d t t^{b}(1-t)^{a}(1+t)^{a+N} .
\end{aligned}
$$

Similarly, for the $(1 \rightarrow \infty)$ channel, one gets

$$
\begin{aligned}
I_{(1 \rightarrow \infty)} & =\int_{1}^{\infty} d y y^{a}(y-1)^{b}(1+y)^{c} \\
& =2^{-2 a-1-N} \int_{0}^{1} d t t^{b}(1-t)^{a+N}(1+t)^{a} .
\end{aligned}
$$


The sum of the two channels gives

$$
\begin{aligned}
I & =I_{(0 \rightarrow 1)}+I_{(1 \rightarrow \infty)} \\
& =2^{-2 a-1-N} \int_{0}^{1} d t t^{b}(1-t)^{a}(1+t)^{a}\left[(1+t)^{N}+(1-t)^{N}\right] \\
& =2^{-2 a-1-N} \sum_{m=0}^{N}\left[1+(-1)^{m}\right]\left(\begin{array}{l}
N \\
m
\end{array}\right) \int_{0}^{1} d t t^{b+m}(1-t)^{a}(1+t)^{a} \\
& =2^{-2 a-2-N} \sum_{m=0}^{N}\left[1+(-1)^{m}\right]\left(\begin{array}{l}
N \\
m
\end{array}\right) \cdot \frac{\Gamma(a+1) \Gamma\left(\frac{b+1}{2}+\frac{m}{2}\right)}{\Gamma\left(a+\frac{b+3}{2}+\frac{m}{2}\right)} \\
& =2^{-2 a-1-N} \frac{\Gamma(a+1) \Gamma\left(\frac{b+1}{2}\right)}{\Gamma\left(a+\frac{b+3}{2}\right)} \sum_{n=0}^{\left[\frac{N}{2}\right]}\left(\begin{array}{l}
N \\
2 n
\end{array}\right) \frac{\left(\frac{b+1}{2}\right)_{n}}{\left(a+\frac{b+3}{2}\right)_{n}} \\
& =2^{-2 a-1-N} \cdot B\left(a+1, \frac{b+1}{2}\right) \cdot{ }_{3} F_{2}\left(\frac{b+1}{2},-\left[\frac{N}{2}\right], \frac{1}{2}-\left[\frac{N}{2}\right] ; a+\frac{b+3}{2}, \frac{1}{2} ; 1\right)
\end{aligned}
$$

where the generalized hypergeometric function ${ }_{3} F_{2}$ is defined in the appendix. Note that the energy dependence of the prefactor $4(2 i)^{k_{1} \cdot D \cdot k_{1}+k_{2} \cdot D \cdot k_{2}}$ in the scattering amplitude cancels, apart from an irrelvant factor, the energy dependence of $2^{-2 a-1-N}$ by using Eq.(2.26). For $N=0$, one recovers the result of tachyon scattering amplitude Eq.(2.10). For the special arguments of ${ }_{3} F_{2}$ in Eq.(2.29), the hypergeometric function terminates to a finite sum and, as a result, the whole scattering amplitudes consistently reduce to the usual beta function. The explicit forms of ${ }_{3} F_{2}$ for some integer $N$ are given in the appendix.

\section{HIGH ENERGY SCATTERING AMPLITUDES}

In this section, we will calculate the high energy limit of string scattered from D-brane. In particular, we will calculate the ratios among scattering amplitudes of different string states at high energies. We first begin with a brief review of the calculation of these ratios without D-brane. There are three methods to calculate these ratios [8, 9]. We will only review the method of decoupling of high-energy ZNS for 26D bosonic open string theory. The same ratios can be obtained by two other methods, the high energy Virasoro constraints and the saddle-point method. Since the calculation of decoupling of high energy ZNS without D-brane remains the same as the calculation of string scatters from D-brane, the ratios of these high-energy scattering amplitudes can be used to check the ratios we will obtain for string scatters from D-brane. At a fixed mass level $M_{o p}^{2}=2(n-1)$, it was shown that 
[8, 9] a four-point function is at the leading order in high-energy limit only for states of the following form ( we use the notation of [18])

$$
|n, 2 m, q\rangle \equiv\left(\alpha_{-1}^{T}\right)^{n-2 m-2 q}\left(\alpha_{-1}^{L}\right)^{2 m}\left(\alpha_{-2}^{L}\right)^{q}|0, k\rangle
$$

where $n \geqslant 2 m+2 q, m, q \geqslant 0$. The state in Eq.(3.1) is arbitrarily chosen to be the second vertex of the four-point function. The other three points can be any string states. We have defined the normalized polarization vectors of the second string state to be [ $\underline{5}, \underline{6}]$

$$
\begin{gathered}
e_{P}=\frac{1}{M_{o p}}\left(E_{2}, \mathrm{k}_{2}, 0\right)=\frac{k_{2}}{M_{o p}} \\
e_{L}=\frac{1}{M_{o p}}\left(\mathrm{k}_{2}, E_{2}, 0\right) \\
e_{T}=(0,0,1)
\end{gathered}
$$

in the CM frame contained in the plane of scattering. In the OCFQ spectrum of open bosonic string theory, the solutions of physical states conditions include positive-norm propagating states and two types of zero-norm states. The latter are [18]

$$
\text { Type I : } L_{-1}|x\rangle \text {, where } L_{1}|x\rangle=L_{2}|x\rangle=0, L_{0}|x\rangle=0
$$

$$
\text { Type II : }\left(L_{-2}+\frac{3}{2} L_{-1}^{2}\right)|\widetilde{x}\rangle \text {, where } L_{1}|\widetilde{x}\rangle=L_{2}|\widetilde{x}\rangle=0,\left(L_{0}+1\right)|\widetilde{x}\rangle=0 \text {. }
$$

While Type I states have zero-norm at any space-time dimension, Type II states have zeronorm only at $\mathrm{D}=26$. The decoupling of the following Type I high-energy zero-norm states (HZNS)

$$
L_{-1}|n-1,2 m-1, q\rangle=M_{o p}|n, 2 m, q\rangle+(2 m-1)|n, 2 m-2, q+1\rangle
$$

gives the first high-energy Ward identities

$$
\mathcal{T}^{(n, 2 m, q)}=\left(-\frac{2 m-1}{M_{o p}}\right) \cdots\left(-\frac{3}{M_{o p}}\right)\left(-\frac{1}{M_{o p}}\right) \mathcal{T}^{(n, 0, q+m)} .
$$

where $\mathcal{T}^{(n, 2 m, q)}$ represents the four-point functions at level $n$. Similarly, the decoupling of the following Type II HZNS

$$
L_{-2}|n-2,0, q\rangle=\frac{1}{2}|n, 0, q\rangle+M_{o p}|n, 0, q+1\rangle
$$


gives the second high-energy Ward identities

$$
\mathcal{T}^{(n, 0, q)}=\left(-\frac{1}{2 M_{o p}}\right)^{q} \mathcal{T}^{(n, 0,0)} .
$$

Combining Eqs.(3.8) and (3.10) gives the master formula [8, 9, 10]

$$
\mathcal{T}^{(n, 2 m, q)}=\left(-\frac{1}{M_{o p}}\right)^{2 m+q}\left(\frac{1}{2}\right)^{m+q}(2 m-1) ! ! \mathcal{T}^{(n, 0,0)},
$$

which shows that there is only one independent high-energy scattering amplitudes at each fixed mass level. Eq.(3.11) also gives the ratios of high energy scattering amplitudes among different string states. For the case of closed string, the ratios are the tensor products of two open string ratios [12]. It is interesting to note that [8] in calculating the type II high energy Ward identities Eq.(3.9), we have omitted the second term $\frac{3}{2} L_{-1}^{2}|\widetilde{x}\rangle$ of type II ZNS in Eq.(3.6). It turns out that this omition will not, in the high energy limit, affect the final result.

We now turn to the case of D-brane scatterings. For our purpose here, for simplicity, we will only consider the case with $m=0$. That is, states in Eq.(3.1) without $\left(\alpha_{-1}^{L}\right)^{2 m}$ component. The reason is as following. It was shown that [5, 6, 6] the leading order amplitudes containing this component will drop from energy order $E^{4 m}$ to $E^{2 m}$, and one needs to calculate the complicated naive subleading contraction terms between $\partial X$ and $\partial X$ for the multi-tensor scattering in order to get the real leading order scattering amplitudes. For our closed string scattering calculation here, even for the case of one tachyon and one tensor scattering, one encounters the similar complicated nonzero contraction terms in Eq.(2.5) due to the D-brane. So we will omit high energy scattering amplitudes of string states containing this $\left(\alpha_{-1}^{L}\right)^{2 m}$ component. On the other hand, we will also need the result that the high energy closed string ratios are the tensor product of two pieces of open string ratios [12].

To simplify the kinematics, we consider the case of D-0 brane or D-particle scatterings. The momentum of the incident particle $k_{2}$ is along the $-X$ direction and particle $k_{1}$ is scattered at an angle $\phi$. We will consider the general case of an incoming tensor state $\left(\alpha_{-1}^{T}\right)^{n-2 q}\left(\alpha_{-2}^{L}\right)^{q} \otimes\left(\tilde{\alpha}_{-1}^{T}\right)^{n-2 q^{\prime}}\left(\tilde{\alpha}_{-2}^{L}\right)^{q^{\prime}}|0\rangle$ and an outgoing tachyon state. Our result can be 
easily generalized to the more general two tensor cases. The kinematic setup is

$$
\begin{aligned}
e^{P} & =\frac{1}{M}\left(-E,-\mathrm{k}_{2}, 0\right)=\frac{k_{2}}{M}, \\
e^{L} & =\frac{1}{M}\left(-\mathrm{k}_{2},-E, 0\right), \\
e^{T} & =(0,0,1), \\
k_{1} & =\left(E, \mathrm{k}_{1} \cos \phi,-\mathrm{k}_{1} \sin \phi\right), \\
k_{2} & =\left(-E,-\mathrm{k}_{2}, 0\right) .
\end{aligned}
$$

For the scattering of D-particle $D_{i j}=-\delta_{i j}$, and it is easy to calculate

$$
\begin{aligned}
e^{T} \cdot k_{2} & =e^{L} \cdot k_{2}=0, \\
e^{T} \cdot k_{1} & =-\mathrm{k}_{1} \sin \phi \sim-E \sin \phi, \\
e^{T} \cdot D \cdot k_{1} & =\mathrm{k}_{1} \sin \phi \sim E \sin \phi, \\
e^{T} \cdot D \cdot k_{2} & =0, \\
e^{L} \cdot k_{1} & =\frac{1}{M}\left[\mathrm{k}_{2} E-\mathrm{k}_{1} E \cos \phi\right] \sim \frac{E^{2}}{M}(1-\cos \phi), \\
e^{L} \cdot D \cdot k_{1} & =\frac{1}{M}\left[\mathrm{k}_{2} E+\mathrm{k}_{1} E \cos \phi\right] \sim \frac{E^{2}}{M}(1+\cos \phi), \\
e^{L} \cdot D \cdot k_{2} & =\frac{1}{M}\left[-\mathrm{k}_{2} E-\mathrm{k}_{2} E\right] \sim-\frac{2 E^{2}}{M},
\end{aligned}
$$

and

$$
\begin{aligned}
& a_{0}=k_{1} \cdot D \cdot k_{1}=-E^{2}-\mathrm{k}_{1}^{2} \sim-2 E^{2}, \\
& b_{0}=2 k_{1} \cdot k_{2}+1=2\left(E^{2}-\mathrm{k}_{1} \mathrm{k}_{2} \cos \phi\right)+1 \sim 2 E^{2}(1-\cos \phi), \\
& c_{0}=2 k_{1} \cdot D \cdot k_{2}+1=2\left(E^{2}+\mathrm{k}_{1} \mathrm{k}_{2} \cos \phi\right)+1 \sim 2 E^{2}(1+\cos \phi) .
\end{aligned}
$$

The scattering amplitude is then calculated to be 


$$
\begin{aligned}
A_{\text {tensor }}= & \varepsilon_{T^{n-2 q} L^{q}, T^{n-2 q^{\prime}} L^{q^{\prime}}} \int d^{2} z_{1} d^{2} z_{2}\left\langle V_{1}\left(z_{1}, \bar{z}_{1}\right) V_{2}^{T^{n-2 q} L^{q}, T^{n-2 q^{\prime}} L^{q^{\prime}}}\left(z_{2}, \bar{z}_{2}\right)\right\rangle \\
& =\varepsilon_{T^{n-2 q} L^{q}, T^{n-2 q^{\prime}} L^{q^{\prime}}} \int d^{2} z_{1} d^{2} z_{2} \cdot\left\langle e^{i k_{1} X}\left(z_{1}\right) e^{i k_{1} \tilde{X}}\left(\bar{z}_{1}\right)\right. \\
& \left.\left(\partial X^{T}\right)^{n-2 q}\left(i \partial^{2} X^{L}\right)^{q} e^{i k_{2} X}\left(z_{2}\right)\left(\bar{\partial} \tilde{X}^{T}\right)^{n-2 q^{\prime}}\left(i \bar{\partial}^{2} \tilde{X}^{L}\right)^{q^{\prime}} e^{i k_{2} \tilde{X}}\left(\bar{z}_{2}\right)\right\rangle \\
= & (-1)^{q+q^{\prime}} \int d^{2} z_{1} d^{2} z_{2}\left(z_{1}-\bar{z}_{1}\right)^{k_{1} \cdot D \cdot k_{1}}\left(z_{2}-\bar{z}_{2}\right)^{k_{2} \cdot D \cdot k_{2}}\left|z_{1}-z_{2}\right|^{2 k_{1} \cdot k_{2}}\left|z_{1}-\bar{z}_{2}\right|^{2 k_{1} \cdot D \cdot k_{2}} \\
& \cdot\left[\frac{i e^{T} \cdot k_{1}}{z_{1}-z_{2}}+\frac{i e^{T} \cdot D \cdot k_{1}}{\bar{z}_{1}-z_{2}}+\frac{i e^{T} \cdot D \cdot k_{2}}{\bar{z}_{2}-z_{2}}\right]^{n-2 q} \\
& \left.\cdot \frac{i e^{T} \cdot D \cdot k_{1}}{z_{1}-\bar{z}_{2}}+\frac{i e^{T} \cdot k_{1}}{\bar{z}_{1}-\bar{z}_{2}}+\frac{i e^{T} \cdot D \cdot k_{2}}{z_{2}-\bar{z}_{2}}\right]^{n-2 q^{\prime}} \\
& \cdot\left[\frac{e^{L} \cdot k_{1}}{\left(z_{1}-z_{2}\right)^{2}}+\frac{e^{L} \cdot D \cdot k_{1}}{\left(\bar{z}_{1}-z_{2}\right)^{2}}+\frac{e^{L} \cdot D \cdot k_{2}}{\left(\bar{z}_{2}-z_{2}\right)^{2}}\right]^{q} \\
& \cdot\left[\frac{e^{L} \cdot D \cdot k_{1}}{\left(z_{1}-\bar{z}_{2}\right)^{2}}+\frac{e^{L} \cdot k_{1}}{\left(\bar{z}_{1}-\bar{z}_{2}\right)^{2}}+\frac{e^{L} \cdot D \cdot k_{2}}{\left(z_{2}-\bar{z}_{2}\right)^{2}}\right]^{q^{\prime}} \cdot
\end{aligned}
$$


Set $z_{1}=i y$ and $z_{2}=i$ to fix the $S L(2, R)$ invariance, we have

$$
\begin{aligned}
& A_{\text {tensor }}^{(0 \rightarrow 1)}=4(2 i)^{k_{1} \cdot D \cdot k_{1}+k_{2} \cdot D \cdot k_{2}} \int_{0}^{1} d y y^{k_{1} \cdot D \cdot k_{1}}(1-y)^{2 k_{1} \cdot k_{2}+1}(1+y)^{2 k_{1} \cdot D \cdot k_{2}+1} \\
& \cdot\left[-\frac{e^{T} \cdot k_{1}}{1-y}-\frac{e^{T} \cdot D \cdot k_{1}}{1+y}-\frac{e^{T} \cdot D \cdot k_{2}}{2}\right]^{n-2 q} \\
& \cdot\left[\frac{e^{T} \cdot D \cdot k_{1}}{1+y}+\frac{e^{T} \cdot k_{1}}{1-y}+\frac{e^{T} \cdot D \cdot k_{2}}{2}\right]^{n-2 q^{\prime}} \\
& \cdot\left[\frac{e^{L} \cdot k_{1}}{(1-y)^{2}}+\frac{e^{L} \cdot D \cdot k_{1}}{(1+y)^{2}}+\frac{e^{L} \cdot D \cdot k_{2}}{4}\right]^{q} \\
& \cdot\left[\frac{e^{L} \cdot D \cdot k_{1}}{(1+y)^{2}}+\frac{e^{L} \cdot k_{1}}{(1-y)^{2}}+\frac{e^{L} \cdot D \cdot k_{2}}{4}\right]^{q^{\prime}} \\
& =(-1)^{n} 4(2 i)^{k_{1} \cdot D \cdot k_{1}+k_{2} \cdot D \cdot k_{2}}(E \sin \phi)^{2 n-2\left(q+q^{\prime}\right)}\left(\frac{E^{2}}{M}\right)^{q+q^{\prime}} \\
& \cdot \int_{0}^{1} d y y^{k_{1} \cdot D \cdot k_{1}}(1-y)^{2 k_{1} \cdot k_{2}+1}(1+y)^{2 k_{1} \cdot D \cdot k_{2}+1} \\
& \cdot\left[\frac{1}{1-y}-\frac{1}{1+y}\right]^{2 n-2\left(q+q^{\prime}\right)} \cdot\left[\frac{1-\cos \phi}{(1-y)^{2}}+\frac{1+\cos \phi}{(1+y)^{2}}-\frac{1}{2}\right]^{q+q^{\prime}} \\
& =(-1)^{n} 4(2 i)^{k_{1} \cdot D \cdot k_{1}+k_{2} \cdot D \cdot k_{2}}(2 E \sin \phi)^{2 n}\left(-\frac{1}{8 M \sin ^{2} \phi}\right)^{q+q^{\prime}} \\
& \cdot \sum_{i=0}^{q+q^{\prime}} \sum_{j=0}^{i}\left(\begin{array}{c}
q+q^{\prime} \\
i
\end{array}\right)\left(\begin{array}{l}
i \\
j
\end{array}\right)(-2)^{i}(1-\cos \phi)^{j}(1+\cos \phi)^{i-j} \\
& \int_{0}^{1} d y y^{k_{1} \cdot D \cdot k_{1}}(1-y)^{2 k_{1} \cdot k_{2}+1}(1+y)^{2 k_{1} \cdot D \cdot k_{2}+1} \\
& \cdot\left[\frac{y}{(1-y)(1+y)}\right]^{2 n-2\left(q+q^{\prime}\right)}\left[\frac{1}{1-y}\right]^{2 j}\left[\frac{1}{1+y}\right]^{2(i-j)} .
\end{aligned}
$$

Now in the high energy limit, the master formula Eq.(2.29) reduces to

$$
\begin{aligned}
I & =I_{(0 \rightarrow 1)}+I_{(1 \rightarrow \infty)} \\
& \simeq 2^{-2 a-2-N} B\left(a+1, \frac{b+1}{2}\right)\left[\left(1+\sqrt{\left|\frac{b}{2 a+b}\right|}\right)^{N}+\left(1-\sqrt{\left|\frac{b}{2 a+b}\right|}\right)^{N}\right] \\
& \equiv 2^{-2 a-2-N} B\left(a+1, \frac{b+1}{2}\right) F_{N},
\end{aligned}
$$


where

$$
\begin{aligned}
n_{a} & =2 n-2\left(q+q^{\prime}\right), \\
n_{b} & =-2 n+2\left(q+q^{\prime}\right)-2 j, \\
n_{c} & =-2 n+2\left(q+q^{\prime}\right)-2(i-j), \\
N & =-\left(2 n_{a}+n_{b}+n_{c}\right)=2 i \\
2 a_{0} & =k_{1} \cdot D \cdot k_{1}+k_{2} \cdot D \cdot k_{2}, \\
F_{N} & =\left(1+\sqrt{\frac{1-\cos \phi}{1+\cos \phi}}\right)^{N}+\left(1-\sqrt{\frac{1-\cos \phi}{1+\cos \phi}}\right)^{N} .
\end{aligned}
$$

The total high energy scattering amplitude can then be calculated to be

$$
\begin{aligned}
& A_{\text {tensor }}=A_{\text {tensor }}^{(0 \rightarrow 1)}+A_{\text {tensor }}^{(1 \rightarrow \infty)} \\
& \simeq(-1)^{n} 4(2 i)^{2 a_{0}}(2 E \sin \phi)^{2 n}\left(-\frac{1}{8 M \sin ^{2} \phi}\right)^{q+q^{\prime}} \\
& \cdot \sum_{i=0}^{q+q^{\prime}} \sum_{j=0}^{i}\left(\begin{array}{c}
q+q^{\prime} \\
i
\end{array}\right)\left(\begin{array}{c}
i \\
j
\end{array}\right)(-2)^{i}(1-\cos \phi)^{j}(1+\cos \phi)^{i-j} \cdot 2^{-2 a-2-N} B\left(a+1, \frac{b+1}{2}\right) F_{N} .
\end{aligned}
$$

The high energy limit of the beta function is

$$
B\left(a+1, \frac{b+1}{2}\right) \simeq B\left(a_{0}+1, \frac{b_{0}+1}{2}\right) \frac{a_{0}^{n_{a}}\left(\frac{b_{0}}{2}\right)^{n_{b} / 2}}{\left(a_{0}+\frac{b_{0}}{2}\right)^{n_{a}+n_{b} / 2}}
$$

Finally we get the scattering amplitudes at mass level $M^{2}=2(n-1)$

$$
\begin{aligned}
A_{\text {tensor }} & =A_{\text {tensor }}^{(0 \rightarrow 1)}+A_{\text {tensor }}^{(1 \rightarrow \infty)} \\
& =(-1)^{a_{0}} E^{2 n}\left(-\frac{1}{2 M}\right)^{q+q^{\prime}} B\left(a_{0}+1, \frac{b_{0}+1}{2}\right) \\
& \cdot \sum_{i=0}^{q+q^{\prime}} \sum_{j=0}^{i}\left(\begin{array}{c}
q+q^{\prime} \\
i
\end{array}\right)\left(\begin{array}{c}
i \\
j
\end{array}\right)(-2)^{-i}(1+\cos \phi)^{i}(-1)^{j} F_{N} \\
& =2(-1)^{a_{0}} E^{2 n}\left(-\frac{1}{2 M}\right)^{q+q^{\prime}} B\left(a_{0}+1, \frac{b_{0}+1}{2}\right),
\end{aligned}
$$

where the high energy limit of $B\left(a_{0}+1, \frac{b_{0}+1}{2}\right)$ is independent of $q+q^{\prime}$. We thus have explicitly shown that there is only one independent high energy scattering amplitude at 
each fixed mass level. It is a remarkable result that the ratios $\left(-\frac{1}{2 M}\right)^{q+q^{\prime}}$ for different high energy scattering amplitudes at each fixed mass level is consistent with Eq.(3.11) for the scattering without D-brane as expected. For the two tensor scatterings, the ratios are $\Sigma_{i=1}^{2}\left(-\frac{1}{2 M_{i}}\right)^{\left(q_{i}+q_{i}^{\prime}\right)}$.

\section{CONCLUSION}

In this paper, we have shown that the $(0 \rightarrow 1)$ and $(1 \rightarrow \infty)$ channels scattering amplitudes of an aribitrary closed string state scattered from D-brane to another arbitrary closed string state can be expressed in terms of the hypergeometric function ${ }_{2} F_{1}$, which in turn can be reduced to the usual beta function. We have noted that, mathematically, the reduction of hypergeometric function ${ }_{2} F_{1}$ to the beta function in the string scattering amplitudes is mainly due to the Kummer condition Eq.(2.22). Physically, this condition is realized in the equation of momentum conservation on the D-brane Eq.(2.17). After summing up the $(0 \rightarrow 1)$ and $(1 \rightarrow \infty)$ channels, we discover that all the scattering amplitudes can be expressed in terms of the generalized hypergeometric function ${ }_{3} F_{2}$ with special arguments, which terminates to a finite sum and, as a result, the whole scattering amplitudes consistently reduce to the usual beta function. Our results suggest the interesting relation between string scattering amplitudes and the (generalized) hypergeometric functions both physically and mathematically.

Finally, we explicitly calculate high-energy limit of a series of the above scattering amplitudes for arbitrary mass levels, and derive the linear relations among them for the case of D-particle. Since the calculation of decoupling of high energy ZNS in D-brane scatterings remains the same as the case of scatterings without D-brane, the ratios of these high-energy scattering amplitudes at each fixed mass level are found to be consistent with the calculations of our previous works without D-brane. Presumably, this result can be generalized to the case of general D p-brane except D-instanton and Domain-wall [19].

\section{ACKNOWLEDGMENTS}

This work is supported in part by the National Science Council and National Center of Theoretical Science, Taiwan, R.O.C. 


\section{APPENDIX A: A BRIEF REVIEW OF ${ }_{2} F_{1}$ AND ${ }_{3} F_{2}$}

In this appendix, we review the definitions and some formulas of hypergeometric function ${ }_{2} F_{1}$ and generalized hypergeometric function ${ }_{3} F_{2}$ which we used in the text. hypergeometric functions form an important class of special functions. Many elementary special functions are special cases of ${ }_{2} F_{1}$. The hypergeometric function ${ }_{2} F_{1}$ is defined to be $(\alpha, \beta, \gamma$ constant $)$

$$
\begin{aligned}
{ }_{2} F_{1}(\alpha, \beta, \gamma ; x) & =1+\frac{\alpha \beta}{\gamma} \frac{x}{1 !}+\frac{\alpha(\alpha+1) \beta(\beta+1)}{\gamma(\gamma+1)} \frac{x^{2}}{2 !}+\cdots=\sum_{n=0}^{\infty} \frac{(\alpha)_{n}(\beta)_{n}}{(\gamma)_{n}} \frac{x^{n}}{n !} \\
& =\frac{\Gamma(\gamma)}{\Gamma(\alpha) \Gamma(\beta)} \sum_{n=0}^{\infty} \frac{\Gamma(\alpha+n) \Gamma(\beta+n)}{\Gamma(\gamma+n)} \frac{x^{n}}{n !}
\end{aligned}
$$

where

$$
(\alpha)_{0}=1,(\alpha)_{n}=\alpha(\alpha+1)(\alpha+2) \cdots(\alpha+n-1)=\frac{(\alpha+n-1) !}{(\alpha-1) !} .
$$

The hypergeometric function ${ }_{2} F_{1}$ is a solution, at the singular point $x=0$ with indicial root $r=0$, of the Gauss's hypergeometric differential equation

$$
x(1-x) u^{\prime \prime}+[\gamma-(\alpha+\beta+1)] u^{\prime}-\alpha \beta u=0,
$$

which contains three regular singularities $x=0,1, \infty$. The second solution of Eq. (A.3) with indicial root $r=1-\gamma$ can be expressed in terms of ${ }_{2} F_{1}$ as following $(\gamma \neq$ integer $)$

$$
u_{2}(x)=x^{1-\gamma} 2 F_{1}(\alpha-\gamma+1, \beta-\gamma+1,2-\gamma, x)
$$

Other solutions of Eq. (A.3), which corresponds to singularities $x=1, \infty$, can also be expressed in terms of the hypergeometric function ${ }_{2} F_{1}$. The following identity

$$
{ }_{2} F_{1}(\alpha, \beta, \gamma ; x)=(1-x)^{\gamma-\alpha-\beta}{ }_{2} F_{1}(\gamma-\alpha, \gamma-\beta, \gamma ; x)
$$

which we used in the text can then be derived.

${ }_{2} F_{1}$ has an intergal representation

$$
{ }_{2} F_{1}(\alpha, \beta, \gamma ; x)=\frac{\Gamma(\gamma)}{\Gamma(\beta) \Gamma(\gamma-\beta)} \int_{0}^{1} d y y^{\beta-1}(1-y)^{\gamma-\beta-1}(1-y x)^{-\alpha}
$$

which can be used to do analytic continuation. Equation (A.6) with $x=-1$ was repeatedly used in the text in our calculations of string scattering amplitudes with D-brane. 
There exists interesting relations among hypergeometric function ${ }_{2} F_{1}$ with different arguments

$$
x^{-p}(1-x)^{-q} 2 F_{1}(\alpha, \beta, \gamma ; x)=t^{-p^{\prime}}(1-t)_{2}^{-q^{\prime}} F_{1}\left(\alpha^{\prime}, \beta^{\prime}, \gamma^{\prime} ; t\right),
$$

where $x=\varphi(t)$ is an algebraic function with degree up to six. As an example, the quadratic transformation formula

$$
{ }_{2} F_{1}(\alpha, \beta, 1+\alpha-\beta ; x)=(1-x)^{-\alpha}{ }_{2} F_{1}\left(\frac{\alpha}{2}, \frac{1+\alpha-2 \beta}{2}, 1+\alpha-\beta ; \frac{-4 x}{(1-x)^{2}}\right),
$$

can be used to derive the Kummer's relation

$$
{ }_{2} F_{1}(\alpha, \beta, 1+\alpha-\beta,-1)=\frac{\Gamma(1+\alpha-\beta) \Gamma\left(1+\frac{\alpha}{2}\right)}{\Gamma(1+\alpha) \Gamma\left(1+\frac{\alpha}{2}-\beta\right)},
$$

which is crucial to reduce the scattering amplitudes of string from D-brane to the usual beta function.

In summing up the $(0 \rightarrow 1)$ and $(1 \rightarrow \infty)$ channel scattering amplitudes, we have used the master formula

$$
\begin{aligned}
I & =I_{(0 \rightarrow 1)}+I_{(1 \rightarrow \infty)} \\
& =2^{-2 a-1-N} \int_{0}^{1} d t t^{b}(1-t)^{a}(1+t)^{a}\left[(1+t)^{N}+(1-t)^{N}\right] \\
& =2^{-2 a-1-N} \sum_{n=0}^{\left[\frac{N}{2}\right]}\left(\begin{array}{c}
N \\
2 n
\end{array}\right) \cdot B\left(a+1, \frac{b+1}{2}+n\right) \\
& =2^{-2 a-1-N} \cdot B\left(a+1, \frac{b+1}{2}\right) \cdot{ }_{3} F_{2}\left(\frac{b+1}{2},-\left[\frac{N}{2}\right], \frac{1}{2}-\left[\frac{N}{2}\right] ; a+\frac{b+3}{2}, \frac{1}{2} ; 1\right) .
\end{aligned}
$$

In Equation (A.10), $B$ is the beta function and ${ }_{3} F_{2}$ is the generalized hypergeometric function, which is defined to be

$$
{ }_{3} F_{2}\left(\alpha_{1}, \alpha_{2}, \alpha_{3} ; \gamma_{1}, \gamma_{2} ; x\right)=\sum_{n=0}^{\infty} \frac{\left(\alpha_{1}\right)_{n}\left(\alpha_{2}\right)_{n}\left(\alpha_{3}\right)_{n}}{\left(\gamma_{1}\right)_{n}\left(\gamma_{2}\right)_{n}} \frac{x^{n}}{n !} .
$$

For those arguments of ${ }_{3} F_{2}$ in Eq. (A.10), the series of the generalized hypergeometric 
function ${ }_{3} F_{2}$ terminates to a finite sum. For example,

$$
\begin{aligned}
& N=0:{ }_{3} F_{2}=1, \\
& N=1:{ }_{3} F_{2}=1, \\
& N=2:{ }_{3} F_{2}=\frac{a+b+2}{a+\frac{b+3}{2}}, \\
& N=3:{ }_{3} F_{2}=\frac{a+2 b+3}{a+\frac{b+3}{2}}, \\
& N=4:{ }_{3} F_{2}=\frac{a^{2}+4 a b+2 b^{2}+7 a+12 b+12}{\left(a+\frac{b+3}{2}\right)\left(a+\frac{b+5}{2}\right)}, \\
& N=5:{ }_{3} F_{2}=\frac{a^{2}+6 a b+9 b^{2}+4 a+22 b+20}{\left(a+\frac{b+3}{2}\right)\left(a+\frac{b+5}{2}\right)} .
\end{aligned}
$$

[1] F.Liu, Phys. Rev. D38,1334 (1988). J. C. Lee, Prog. Theor. Phys. 91, 353 (1994); Phys. Lett. B 337, 69 (1994);

[2] D. J. Gross and P. F. Mende, Phys. Lett. B 197, 129 (1987); Nucl. Phys. B 303, 407 (1988).

[3] D. J. Gross, Phys. Rev. Lett. 60, 1229 (1988); Phil. Trans. R. Soc. Lond. A329, 401 (1989).

[4] D. J. Gross and J. L. Manes, Nucl. Phys. B 326, 73 (1989). See section 6 for details.

[5] C. T. Chan and J. C. Lee, Phys. Lett. B 611, 193 (2005). J. C. Lee, arXiv:hep-th/0303012].

[6] C. T. Chan and J. C. Lee, Nucl. Phys. B 690, 3 (2004).

[7] C. T. Chan, P. M. Ho and J. C. Lee, Nucl. Phys. B 708, 99 (2005).

[8] C. T. Chan, P. M. Ho, J. C. Lee, S. Teraguchi and Y. Yang, Nucl. Phys. B 725, 352 (2005).

[9] C. T. Chan, P. M. Ho, J. C. Lee, S. Teraguchi and Y. Yang, Phys. Rev. Lett. 96 (2006) 171601.

[10] C. T. Chan, P. M. Ho, J. C. Lee, S. Teraguchi and Y. Yang, Nucl. Phys. B 749, 266 (2006). "Comments on the high energy limit of bosonic open string theory," arXiv:hep-th/0509009.

[11] C. T. Chan, J. C. Lee and Y. Yang, Nucl. Phys. B 738, 93 (2006).

[12] C. T. Chan, J. C. Lee and Y. Yang, Nucl. Phys. B 749, 280 (2006).

[13] Pei-Ming Ho, Xue-Yan Lin, Phys.Rev. D73 (2006) 126007.

[14] J. C. Lee, Phys. Lett. B 241, 336 (1990); Phys. Rev. Lett. 64, 1636 (1990). J. C. Lee and B. Ovrut, Nucl. Phys. B 336, 222 (1990), Phys. Lett. B 326, 79 (1994).

[15] T. D. Chung and J. C. Lee, Phys. Lett. B 350, 22 (1995). Z. Phys. C 75, 555 (1997). J. C. Lee, Eur. Phys. J. C 1, 739 (1998). 
[16] H. C. Kao and J. C. Lee, Phys. Rev. D 67, 086003 (2003). . C. T. Chan, J. C. Lee and Y. Yang, Phys. Rev. D 71, 086005 (2005)

[17] For a review, see A. Hashimoto and I.R. Klebanov, "Scattering of strings from D-branes" hep-th/9611214 and references therein. M.R. Garousi and R.C. Myers, "Superstring scattering from D-Branes" Nucl. Phys. B475 (1996) 193, hep-th/9603194. I.R. Klebanov and L. Thorlacius, Phys. Lett. B371,51 (1996). J.L.F. Barbon, Phys. Lett. B382, 60 (1996). C. Bachas and B. Pioline, JHEP9912, 004 (1999). S. Hirano and Y. Kazama, Nucl. Phys. B499, 495 (1997).

[18] M. B. Green, J. H. Schwarz and E. Witten, "Superstring Theory. Vol. 1," Cambridge University Press 1987.

[19] C. T. Chan, J. C. Lee and Y. Yang, "Power-law Behavior of Strings Scattered from Domainwall and Breakdown of Their High Energy Linear Relations", hep-th/0610219. 